\section{Aus der Musiksoziologischen Forschungsstelle}

RENATE MÜLLER

\section{Forschungsprojekte}

\section{Marc Calmbach: „More than Music - eine theoretische und empirische musik- und kultursoziologische Un- tersuchung der Jugendkultur Hard- core $^{\text {c6 }}$}

More than Music ist ein Forschungsprojekt der Musiksoziologischen Forschungsstelle, das von der PH Ludwigsburg gefördert und durch ein Promotionsstipendium nach dem Landesgraduiertenförderungsgesetz unterstützt wird. In seiner Dissertation analysiert Marc Calmbach (http://www.ph-ludwigsburg.de/3231.html) die Jugendkultur Hardcore, in deren Zentrum die alternative Kulturproduktion nach dem Do-ityourself-Prinzip (DIY) steht. Bei DIY geht es darum, Kultur bzw. kulturelle Objekte möglichst unabhängig von kommerziellen Strukturen zu schaffen und sich somit als widerspenstige Alternative zum Mainstream resp. zur Kulturindustrie $\mathrm{zu}$ positionieren und $\mathrm{zu}$ organisieren. DIY-Aktivismus wird in mehrfacher Hinsicht in den Blick genommen:

- DIY wird am Beispiel der Produktion und des Vertriebs von Fanzines und Tonträgern sowie der Organisation von Konzerten als Praxis jugendkultureller Selbstermächtigung bzw. Widerspenstigkeit beschrieben.

- DIY wird hinsichtlich seiner Bedeutung als Authentizitätskriterium in den Blick genommen. Theoretischer Hintergrund sind dabei die Konzepte der kulturellen Ökonomie von Bourdieu, Fiske und Thornton sowie Bourdieus Überlegungen zum Feld künstlerischer Produktion. Über DIY wird demonstratives Desinteresse gegenüber kommerziellen Zielsetzungen, Strategien und Mechanismen symbolisiert. Es wird die These verfolgt, dass das DIY-Prinzip in der Hardcore-Szene als zentraler Gradmesser zur Bestimmung von Authentizität herangezogen wird: Authentisch ist - insbesondere aus Sicht des „harten Kerns" - wer sich in und für die Szene nach dem DIY-Prinzip engagiert und nicht, wer „nur“ nach Hardcore klingt oder aussieht.

- Vor dem Hintergrund der Theorie der musikalischen und medialen Selbstsozialisation sowie dem Konzept der „unsichtbaren Bildungsprogramme“ (Hitzler) wird die Frage der Aneignung und der Vermittlung der entsprechenden Wissensbestände, Diskurse und Kompetenzen beleuchtet, die notwendig sind, um nach dem DIY-Prinzip handeln zu können. Es wird gezeigt, dass die für
$D I Y$ notwendigen Fähigkeiten, Fertigkeiten und Wissensformen außerhalb legitimer Bildungsinstitutionen erworben werden.

Neben der kulturellen Praxis und deren jugendund kultursoziologischen Verortung steht die Analyse des hochdifferenzierten HardcorePublikums im Zentrum der Arbeit. Anhand einer Fragebogenbefragung von 410 Konzertbesuchern auf Clubkonzerten und Festivals in Belgien, Deutschland, Luxemburg, Spanien und Tschechien zwischen Juni und Oktober 2004 wird u. a. die soziokulturelle, musikalische und politische Orientierung des HC-Publikums untersucht. Außerdem wird mit den musikalischen Urteilen des HC-Publikums das ästhetische Urteilsverhalten von Hörern einer Randgruppenmusik beleuchtet. In einer Reihe von Vorträgen auf Kongressen und Tagungen stellten Mitglieder der Musiksoziologischen Forschungsstelle Ergebnisse des Projekts More than Music vor:

- Vortrag „More than Music. Musiksoziologische Einblicke in die Jugendkultur Hardcore" im Doktorandenkolloquium von Prof. Rainer Winter am Institut für Medien- und Kommunikationswissenschaft der Universität Klagenfurt am 08.06.2005.

- Vortrag (mit Stefanie Rhein) „Be part of the scene, not just the scenery - Zur Bedeutung des doit-yourself-Prinzips in der Jugendkultur Hardcore" auf dem Kongress „Landscapes of Cultural Studies“ an der Universität Klagenfurt vom 13.-15.10.2005.

- Vortrag „DIY or DIE. Alternative Kulturproduktion in Jugendszenen" im Rahmen der Kulturveranstaltungen „Montage“ im Café Scholz in Stuttgart am 14.11.2005.

- Vortrag (mit Stefanie Rhein) „DIY OR DIE! - Eine empirische Untersuchung der sozialen und kulturellen Relevanz des do-it-yourself-Prinzips für die Jugendkultur Hardcore " auf der Frühjahrstagung „Arbeit, Politik und Vergnügen in Jugendkulturen“ der Sektionen Jugendsoziologie und Kultursoziologie der Deutschen Gesellschaft für Soziologie an der PH Ludwigsburg im März 2006.

Renate Müller, Stefanie Rhein und Marc Calmbach: "What difference does it make?" Die empirische Ästhetik von The Smiths: Wie gehen Jugendliche mit der Smiths-Ästhetik um? Eine experimentelle audiovisuelle Befragung.

Die empirische Ästhetik von The Smiths ist ein Forschungsprojekt der Musiksoziologischen Forschungsstelle, das von der PH Ludwigsburg und MEDIAN, Gesellschaft für Medien und Analysen e.V. gefördert wurde. Es handelt sich um 
ein Kooperationsprojekt von Dr. Stefanie Rhein (http://www.ph-ludwigsburg.de/3230. html), Marc Calmbach (http://www.phludwigsburg.de/3231.html) und Prof. Dr. Renate Müller (http://www.phludwigsburg.de/3229.html).

Vom 8.-9. April 2005 fand am Manchester Institute of Popular Culture „A Symposium on The Smiths" statt, auf dem neben der kulturellen Bedeutung dieser Popgruppe der 1980er ,ihr außergewöhnliches Vermögen, ihre Zuhörer zu berühren“, wissenschaftlich gewürdigt werden sollte. Dies war Anlass, die $\mathrm{Zu}-$ schreibung sozialer Bedeutung an die mittlerweile 20 Jahre alte Ästhetik von The Smiths vor dem Hintergrund der Theorie des sozialen Gebrauchs von Musik zu untersuchen. Im Zentrum stehen Zusammenhänge zwischen dem Musikgeschmack und der jugendmusikkulturellen Verortung der Befragten auf der einen und der subjektiven Attraktivität der Smiths-Ästhetik sowie dem Umgehen damit (u.a. musikalische Offenheit, mood managing, ästhetische Urteile, soziokulturelle Zuschreibungen an musikalisch Ähnlich- und Andersdenkende) auf der anderen Seite. Anhand eines audiovisuellen Fragebogens wurden Anfang 2005 ca. 170 junge Erwachsene zur Smiths-Ästhetik (Musik, Bilder, Videos) befragt. Die Stichprobe bestand aus Studierenden verschiedener Hochschulen bzw. Fächer (Musik, Medien, Pädagogik, Kulturmanagement), aus jungen Berufstätigen und aus The Smiths-Fans. Die bislang erarbeiteten Ergebnisse konzentrieren sich auf die Zuneigung zur Band The Smiths (11\% The Smiths-Fans, 31\% „Zugeneigte“, 41\% „Indifferente“, 17\% „Abgeneigte") und die damit einhergehenden Distinktionsachsen und ImageZuschreibungen an The Smiths. Die Studie gibt einen Einblick in die Mehrdeutigkeit medial vermittelter populärer Kultur und die damit einhergehende Differenzierung der Bedeutungszuschreibungen an die Ästhetik von The Smiths.

Stefanie Rhein, Marc Calmbach und Renate Müller präsentierten Ergebnisse des Projekts Die empirische Ästhetik von The Smiths:

- Vortrag "What difference does it make? The Empirical Aesthetics Of The Smiths: How Do Young People Relate To The Smiths-Aesthetics? An Experimental Audiovisual Survey" auf dem Symposium "Why Pamper Life's Complexities?" in Manchester im April 2005.

- Vortrag „What difference does it make? Wissenschaftliches zu The Smiths" im Rahmen der Kulturveranstaltungen „Montage“ im Club HI in Stuttgart im Mai 2005.
- Vortrag "Die Empirische Ästhetik von The Smiths" an der PH Ludwigsburg im Juli 2005.

- Vortrag „Empirische Ästhetik - The Smiths. Eine audiovisuelle Studie zur sozialen Bedeutung des Musikgeschmacks" auf der Jahrestagung „Musik und Gedächtnis“ der Deutschen Gesellschaft für Musikpsychologie (DGM) im September 2005 in Würzburg.

- Vortrag „What difference does it make? Die empirische Ästhetik von The Smiths: Wie gehen Jugendliche mit der Smiths-Ästhetik um? Eine experimentelle audiovisuelle Befragung" auf der 16. Arbeitstagung "Konfektionsgröße 5 Schnittmuster populärer Musiken" des Arbeitskreises Studium Populärer Musik (ASPM) im Oktober 2006 in Barendorf.

\section{„Miniforschungsprojekte“ im Rah- men von Lehrveranstaltungen zu präsentativen Forschungsmethoden mit audiovisuellen Fragebögen}

Im Sommersemester 2005 dienten das Lied Die Gerd Show - Im Wablkampf vor mir (Zonenmädchen) und im Wintersemester 2005/ 2006 der Erfolg der Teenieband Tokio Hotel als Ideenlieferanten für „Forschungsetüden“ der Seminare „Studierende forschen mit dem MultiMediaComputer".

- „Abmackern" bringt nichts. Eine experimentelle Studie zum Einfluss des Liedes „Im Wablkampf vor mir (Zonenmädchen) " auf die Images der Kanzlerkandidaten Angela Merkel und Gerbard Schröder.

- „Sieht aus wie ein Mädchen - ist ein Junge“. Sozialästhetisches Umgehen von Grundschülern mit der Band Tokio Hotel. 